\title{
Profil Des Infections Osteo-Articulaires A Germes Banals En Hospitalisation Au Service De Rhumatologie Du Chu De Cocody
}

\author{
Djaha Kouassi Jean-Mermoze \\ Ouattara Baly \\ Massi Gbètondji Michel \\ Hounsounou Mariano \\ Gbané Mariam \\ Diomandé Mohamed \\ Eti Edmond
}

Service de rhumatologie CHU de Cocody, Abidjan, Côte d'Ivoire

\section{Doi:10.19044/esj.2021.v17n29p201}

Submitted: 10 May 2021

Accepted: 01 July 2021

Published: 31 August 2021
Copyright 2021 Author(s)

Under Creative Commons BY-NC-ND

Cite As:

Djaha Kouassi J-M., Ouattara B., Massi Gbètondji M., Hounsounou M., Gbané M., Diomandé M. \& Eti E. (2021). Profil Des Infections Osteo-Articulaires A Germes Banals En Hospitalisation Au Service De Rhumatologie Du Chu De Cocody. European Scientific Journal, ESJ, 17(29), 201.

https://doi.org/10.19044/esj.2021.v17n29p201

\section{Résumé}

Objectif: Déterminer les aspects épidémiologiques, cliniques, étiologiques et thérapeutiques des infections ostéo-articulaires à germes banals.

Matériels et méthode: Il s'est agi d'une étude transversale sur une période de 5 ans allant de janvier 2014 à décembre 2018. Tous les patients hospitalisés dans le service de rhumatologie du CHU de Cocody pour une infection ostéoarticulaire à germe banal ont été inclus dans l'étude. Les données recueillies à l'aide d'une fiche d'enquête ont été analysées grâce au logiciel Epi Info 7.

Résultats: Sur 2046 patients hospitalisés, 71 infections ostéoarticulaires à germes banals ont été recensées soit une fréquence hospitalière de 3,4 \%. Il y'avait 36 femmes et 35 hommes soit un sex-ratio de 0,97. L'âge moyen était de 47,6 ans [25-68 ans]. Les facteurs de risque retrouvés étaient 
le diabète (19,7 \%), l'infection par le VIH (11,2 \%), l'alcool (14,1 \%) et la drépanocytose (8,4\%). Il y'avait une porte d'entrée dans 71,7 \% des cas. Les portes d'entrée étaient cutanées (39,4 \%), urogénitales (28\%), otorhinolaryngologiques (22\%) et bucco dentaires $(10,6 \%)$. Les rachialgies (34\%) et les gonalgies (28\%) représentaient plus de la moitié des motifs de consultations. La fièvre (85,9 \%) était le principal signe général. L’arthrite septique $(59,1 \%)$ et la spondylodiscite à germe banal $(33,8 \%)$ étaient les formes cliniques les plus representées. Les localisations de l'infection prédominaient aux genoux (39,4\%) et au rachis lombaire (23,9\%). La recherche de germe avait été infructueuse dans $84,6 \%$ des cas . Les germes ont été isolés chez seulement 11 patients (15,4\%). Les germes isolés étaient le Staphylocoque aureus dans 36,4 \% des cas. Tous les patients ont eu une double antibiotherapie probabiliste et réajusté secondairement par l'antibiogramme dans $15,4 \%$ des cas. La double antibiothérapie probabiliste était faite de l'association de quinolones-aminoside et de quinolonesbêtalactamines. Les aminosides étaient données sur une durée maximum de 10 jours à cause de l'atteinte rénale. La durée du traitement antibiotique était de 6 semaines en l'absence d'atteinte osseuse et était d'au moins 3 mois en cas d'atteinte osseuse. Il y'avait 07 patients (10\%) qui ont eu une intervention chirurgicale. L'évolution a été favorable chez tous les patients.

Conclusion: Les infections ostéo-articulaires à germes banals sont peu fréquentes en hospitalisation de rhumatologie à Abidjan et touchent les adultes d'âge mur.

Elles se présentent le plus souvent sous forme d'une arthrite septique et d'une spondylodiscite à germe banal. L'évolution était favorable chez tous les patients.

Mots-clés: Arhtrite Septique, Spondylodiscite À Germe Banal, Antibiotique 


\title{
Profile Of Osteo-Articular Infections With Banal Germs In Hospitalization In The Rheumatology Department Of Cocody Chu
}

\author{
Djaha Kouassi Jean-Mermoze \\ Ouattara Baly \\ Massi Gbètondji Michel \\ Hounsounou Mariano \\ Gbané Mariam \\ Diomandé Mohamed \\ Eti Edmond
}

Service de rhumatologie CHU de Cocody, Abidjan, Côte d'Ivoire

\section{Abstract}

Objective: To determine the epidemiological, clinical, etiological and therapeutic aspects of osteo-articular infections caused by banal germs.

Materials and method: This was a cross-sectional study over a 5 year period from January 2014 to December 2018.

All patients hospitalized in the rheumatology department of Cocody University Hospital for an osteoarticular infection caused by germs banals were included in the study. The data collected using a survey sheet was analyzed using Epi Info 7 software.

Results: Out of 2,046 hospitalized patients, 71 osteoarticular infections with banal germs were identified, either a hospital frequency of 3.4 $\%$. There were 36 women and 35 men either a sex ratio of 0.97 . The mean age was 47.6 years [25-68 ans]. The risk factors found were diabetes $(19.7 \%)$, HIV infection (11.2\%), alcohol (14.1\%) and sickle cell disease (8.4 \%). There was a front door $71.7 \%$ of the time. The gates to the front door were cutaneous (39.4\%), urogenital (28\%), otorhinolaryngological (22\%) and oral dentistry (10.6\%). Back pain (34\%) and gonalgia (28\%) accounted for more than half of the reasons for consultations. The main general sign was fever (85.9\%). Septic arthritis (59.1\%) and spondylodiscitis caused by Germs Banals (33.8 $\%)$ were the most common clinical forms. The sites of infection predominated in the knees (39.4\%) and the lumbar spine $(23.9 \%)$. The search for germs was unsuccessful in $84.6 \%$ of cases. The germs were isolated from only 11 patients (15.4\%). The organisms isolated were Staphylococcus aureus in 36.4 $\%$ of cases. All the patients underwent a double probabilistic antibiotherapy and secondarily readjusted by the antibiogram in $15.4 \%$ of cases. The double probabilistic antibiotic therapy consisted of a combination of quinolonesaminoglycoside and quinolones-beta-lactams. Aminoglycosides were given for a short period of up to 10 days because of kidney damage.The duration of 
antibiotic treatment was 6 weeks in the absence of bone involvement and was at least 3 months in the case of bone involvement. There were 07 patients (10 $\%)$ who had surgery. The outcome was favorable in all patients.

Conclusion: Bone and joint infections caused by banal germs are infrequent in rheumatology hospitalizations in Abidjan and affect mature adults. They most often present as septic arthritis and spondylodiscitis with common germs. The outcome was favorable in all patients.

Keywords: Septic Arthritis, Common Germ Spondylodiscitis, Antibiotic

\section{Introduction}

L'infection ostéo-articulaire est une affection sévère surtout lorsqu'elle intéresse le rachis (Valérie \& al, 2014). Les infections ostéoarticulaires demeurent un problème de santé publique en Afrique (Nzenze \& al, 2001; N’tsiba \& al, 2006 ; Ouédraogo \& al, 2014) . Les infections ostéoarticulaires à germes banals constituent une urgence médicale car le pronostic vital est mis en jeu. Le traitement antibiotique doit débuter immédiatement dès la suspicion diagnostique et doit être adapté secondairement à l'antibiotigramme pour éviter les complications pouvant entrainer la mort du patient.

L’objectif de notre travail est de déterminer le profil épidémiologique, clinique, étiologique et thérapeutique des infections ostéoarticulaires à germes banals.

\section{Matériels et méthodes}

Il s'est agit d'une étude transversale sur une période de 5 ans allant de janvier 2014 à décembre 2018. Tous les patients hospitalisés dans le service de rhumatologie du CHU de Cocody à Abidjan en Côte d'Ivoire pour une infection ostéoarticulaire à germe banal ont été inclus dans l'étude. Les patients n'ayant pas un mininum d'examen paraclinique (Numération Formule Sanguine, C réactive protéine, radiographie standard) et les infections ostéoarticulaires tuberculeuses n'ont pas été inclus dans l'étude. Les données étudiées étaient épidémiologiques (âge, sexe), cliniques (les facteurs de risques, le mode de vie, la porte d'entrée, le siège des atteintes, les signes généraux et les signes physiques), paracliniques (la numération formule sanguine, la vitesse de sédimentation, la $\mathrm{C}$ réactive protéine, l'hémoculture, l'analyse du liquide articulaire, l'analyse de la ponction d'abcès, la radiographie standard, l'echographie articulaire, le scanner et l'imagérie par raisonnance magnétique), étiologiques (germes isolés) et thérapeutiques (antibiotique, chirurgie). Les données recueillies à l'aide d'une fiche d'enquête ont été analysées grâce au logiciel Epi Info 7. 


\section{Résultats}

\section{Données socio-démographiques}

Sur 2046 patients hospitalisés, 71 infections ostéo-articulaires à germes banals ont été recensées soit une fréquence hospitalière de 3,4 \%. Il y'avait 36 femmes et 35 hommes soit un sex-ratio de 0,97. L'âge moyen était de 47,6 \pm 18,1 [extrêmes25-68 ] ans.

\section{Données cliniques}

Les facteurs de risque retrouvés étaient le diabète (19,7 \%), l'infection par le VIH (11,2 \%), l'alcool (14,1 \%) et la drépanocytose (8,4 \%). Il y’avait une porte d'entrée dans $71,7 \%$ des cas. Les portes d'entrée étaient cutanées (39,4\%), urogénitales (28\%), otorhinolaryngologiques (22\%) et bucco dentaires (10,6\%). Les rachialgies (34 \%) et les gonalgies (28\%) représentaient plus de la moitié des motifs de consultations. La fièvre (85,9\%) était le principal signe général.

L'arthrite septique (59,1\%) et la spondylodiscite à germe banal (33,8\%) étaient les formes cliniques les plus representées (tableau I).

Tableau I. Répartition des patients selon les formes cliniques des infections

\begin{tabular}{|lcc|}
\hline & Effectif & Pourcentage (\%) \\
\hline Arthrite septique & 42 & 59,1 \\
Spondylodiscite à germe banal & 24 & 33,8 \\
Ostéo-arthrite & 8 & 11,2 \\
Ostéomyélite & 2 & 2,8 \\
Ostéite & 1 & 1,4 \\
\hline
\end{tabular}

Les localisations de l'infection prédominaient aux genoux (39,4 \%) et au rachis lombaire (23,9\%) (tableau II).

\begin{tabular}{|c|c|c|c|}
\hline & & Effectif & Pourcentage (\%) \\
\hline \multirow{11}{*}{$\begin{array}{c}\text { Squelette } \\
\text { périphérique }\end{array}$} & Genou & 28 & 39,4 \\
\hline & Epaule & 9 & 12,6 \\
\hline & Cheville & 7 & 09,8 \\
\hline & Hanche & 7 & 09,8 \\
\hline & Mains & 3 & 04,2 \\
\hline & Poignet & 3 & 04,2 \\
\hline & Pieds & 2 & 2,8 \\
\hline & Coudes & 2 & 2,8 \\
\hline & Jambes & 2 & 2,8 \\
\hline & Cuisse & 1 & 1,4 \\
\hline & Bras & 1 & 1,4 \\
\hline \multirow[t]{4}{*}{ Squelette axial } & Rachis lombaire & 17 & 23,9 \\
\hline & Charnière lombo-sacrée & 7 & 09,8 \\
\hline & Rachis cervical & 6 & 08,4 \\
\hline & Rachis dorsal & 3 & 04,2 \\
\hline
\end{tabular}

Tableau II. Répartition des patients selon le siège de l’infection 


\section{Données paracliniques}

La recherche de germe avait été infructueuse dans $84,6 \%$ des cas .Les germes ont été isolés chez seulement 11 patients (15,4\%). Les germes isolés étaient le staphylocoque aureus dans 36,4 \% des cas (tableau III).

Tableau III. Répartition des patients selon les germes isolés

\begin{tabular}{|lcc|}
\hline & Effectif & Pourcentage (\%) \\
\hline Staphylocoque aureus & 4 & 36,4 \\
Streptocoque SPP & 2 & 18,2 \\
Eschérichia coli & 2 & 18,2 \\
Salmella SPP & 2 & 18,2 \\
Enterococcus facalis & 1 & 9,1 \\
\hline
\end{tabular}

\section{Données thérapeutiques}

Tous les patients ont eu une double antibiotherapie probabiliste et réajusté secondairement par l'antibiogramme dans 15,4\% des cas. La double antibiothérapie probabiliste était faite de l'association de quinolonesaminoside et de quinolones-bêtalactamines. Les aminosides étaient données sur une durée maximum de 10 jours à cause de l'atteinte rénale. La durée du traitement antibiotique était de 6 semaines en l'absence d'atteinte osseuse et était d'au moins 3 mois en cas d'atteinte osseuse . Il y'avait 07 patients (10\%) qui avait eu une intervention chirurgicale. L'évolution a été favorable chez tous les patients.

\section{Discussion}

La fréquence hospitalière était de 3,4\%. Cette fréquence basse a été observée par certains auteurs en Arique subsahérienne (Houzou \& al, 2017 ; Zomaletho \& al, 2014). Il y’avait une prédominance féminime. Houzou \& al, 2017 et Oniankitan \& al, 2011 ont également trouvé une prédominance féminime. Par contre Ledoux \& al, 2012 avait noté une prédominance masculine. L'âge moyen était de 47, 6 ans. L'âge moyen inférieur à 50 ans a été décrite dans la littérature (Ogunlusi \& al, 2006 ; Eti \& al, 2000 ; Houzou \& al, 2013) . Les facteurs de risque étaient le diabète, l'nfection par le VIH, l'alcool et la drépanocytose. Ces mêmes facteurs de risques se retrouvent dans les études d'Oniankitan \& al, 2011, d'Eti \& al, 2000 et de N'tsiba \& al, 2004. Il y'avait une porte d'entrée dans $71,7 \%$ des cas. La porte d'entrée était présente dans $60 \%$ dans l'étude d'Aymeric \& al, 2006. La porte d'entrée cutanée prédominait avec 39,4 \% des cas. Dans l'étude de Dubost \& al, 2004 la porte d'entrée cutanée a également été mis en évidence. La fièvre (85,9 \%) était présente chez la majorité des patients.

L'absence de fièvre chez 14, $1 \%$ des patients pouvait retarder le diagnostic. L'absence de fièvre a été décrite dans l'étude Nolla \& al, 2003.

L'arthrite septique (59,1\%) et la spondylodiscite à germe banal $(33,8$ \%) étaient les formes cliniques les plus representées. Houzou \& al, 2017 avait 
également trouvé une prédominance des spondylodiscites infectieuses et des arthrites infectieuses.

Le germe a été mis en évidence chez seulement 15, 4 \% des patients. Dans l'étude d'Oniankitan \& al, 2011 au Togo, les germes ont été isolés chez seulement $19,7 \%$ des patients.

Les infestions ostéo-articulaires étaient peu documentées en Afrique. Cela pourrait s'expliquer par la faiblesse du plateau technique dans les pays africains. Dans les pays dévéloppés le germe est mis en évidence dans 66 à 86 $\%$ des cas (Schattner \& al,1998). Le staphylocoque aureus representait 36,4 \% des germes isolés. Le Staphylocoque aureus est le germe le plus souvent isolé ( Eder \& al, 2005 ; Favero \& al, 2008 ; Khan \& al, 2013). Le traitement d'attaque necessite une association bactéricide de deux antibiotiques synergiques ( Grados \& al, 2007) comme dans notre étude. Il se fait par voie intraveineuse surtout s'il existe une bactériémie associée pendant 2 à 3 semaines selon l'évolution (Ahmad \& al, 2002). Une intervention chirurgicale a été faite chez $10 \%$ de nos patients. Le drainage percutané permet une meilleure récupération fonctionnelle (Broy \& al,1986 ; Maneiro \& al, 2015).

\section{Conclusion}

Les infections ostéo-articulaires à germes banals sont peu fréquentes en hospitalisation de rhumatologie à Abidjan et touchent les adultes d'âge mur. Elles se présentent le plus souvent sous forme d'une arthrite septique et d'une spondylodiscite à germes banals. L'évolution était favorable chez tous les patients.

\section{References:}

1. Valérie B., Valérie C., Béatrice B. \& Jean-L. (2014). Infection rachidienne: du germe lent au staphylocoque. Rev Rhum monographie; $81: 27-35$.

2. Nzenze JR., Belembaogo E., Magne C., Sanou AS., Coniquet S. \& al. (2001). Panorama des arthropathies inflammatoires à Libreville. Analyse de 57 observations. Med Afr Noire; 48 (10) : 399-402.

3. N’tsiba H., Makosso E., Ngandeu-Singwé M. \& Yala F. (2006). Les arthrites septiques en zone tropicale. Apropos de 176 cas observés à Brazaville. Mali Med; 21 : 49-53.

4. Ouédraogo DD., N’tsiba H., Tiendrébéogo Zabsonré J., Tiéno H., Bokassa \& al. (2014). Clinical spectrum of rheumatologic diseases in departement of rheumatology in Ouagadougou ( Burkina Faso). Clin Rheumat; 33(3) : 385-9.

5. 5-Houzou P., Kakpovi K., Fianyo E., Viwalé ESKT., Komi CT. \& al. (2017). Profil des infestions ostéoarticulaires en consultation 
rhumatologique au CHU de Kara (Togo). Europ Scient Journ; 13 (27) : 251-7.

6. Zomaletho Z., Gounougbé M. \& Avimadjè M. (2014) . Affections rhumatologiques en milieu africain à l'ère du $21^{\text {ème }}$ siècle. Medecine d'Afrique noir; 61 : 449-53.

7. Oniankitan O., Bagayoko Y., Fianyo E., Koffi TV., Kakpovi K., Tagbor KC. \& al. (2011). Arthrites infestieuses en consultation rhumatologique à Lomé. Medecine tropicale; $71: 61-2$.

8. Ledoux JE., Toumadre A., Mathieu S., Mrozek N. \& Soubrier M. (2012). Arthrite septique à bactériologie négative chez l'adulte. Rev Rhum; 79 : 137-41.

9. Ogunlusi JD. ( 2006). Septic arthritis in a Nigerian tertiary hospital . Lowa Orthop Journ; $26:$ 45-7.

10. Eti E., Daboiko JC., Debauly S., Ouali B., Ouattara B., Yao N., Gabla A. \& Kouakou NM. (2000). Arthrites à pyogène des membres au CHU de Cocody. A propos de 79 cas. Rhumatologie; 52 : 18-21.

11. Houzou P., Oniankitan O., Kakpovi K, Koffi-Tessio VES., Tagbor KC., Fianyo E. \& Mijiyawa M. ( 2013). Profil des affections rhumatismales chez 13517 patients ouest africains. Tunis Med; 91 : 16-20.

12. N’tsiba H., Bazebissa R., Lamini N. \& Yala F. (2004). Cent cas d'arthrites septiques du genou en zone intertropicale. Bull Soc Path; 97 : 244-6.

13. Aymeric B., Valérie D., Katel G., Sandrine J. \& Alain S. (2006). Arthrites à streptocoque du groupe B. Revue du rhumatisme; 73 : 7669.

14. Dubost JJ., Soubrier M., Dechamp C., Ristori JM. \& Souvezie B. (2004). Streptocal septic arthritis in adults. A sudy of 55 cases with littérature review joint Bone. Spine; 71 : 303-11.

15. Nolla JM., Gomez-Vaquero C., Corbella X., Ordronez S., GarciaGomez C., Perez A. \& al. (2003). Group B streptococcus ( Streptoccocus agalactiae) Pyogenic arthritis in non-pregnant adults. Medecine [Baltimore]; 82 : 119-28.

16. Schattner A., Kenneth L. \& Vosti K. (1998). Bacterial arthritis due to betahemolytic streptococci of serogroups A, B, C, F and G. Medicine [Baltimore]; 77 : 122-39.

17. Eder L., Zisman D., Rozenbaum M. \& Rosner I. (2005). Clinic al features and etiology of septic arthritis in northen Israel. Rheumatology (Oxford); 44 :1559-63.

18. Favero M., Schiavon F., Riato L., Carraro V. \& Punzi L. ( 2008). Septic arthritis : a 12 years retrospective study in a rheumatological University clinis. Rheumatismo; $60: 260-7$. 
19. Khan FY., Abu-Khattab M., Baagar K., Mohamed SF., Elgendy I., Anand D., Malallah H. \& Sanjay D. (2013). Characteristics of patients with definite septic arthritis at Hamat General Hospital Quatar : a hospital based study from 2006 to 2011. Clin Rhumatol ; 32 : 969-73.

20. Franck G., François-Xavier L., Eric Seneville., Réné-Marc Flipo., Jean-Luc S. \& Patrice F. (2007). Proposition pour la prise en charge thérapeutique des spondylodiscites bactériennes non tuberculeuses de l'adulte. Rev Rhum; 74 : 225-31.

21. Ahmed R., Daoudi Y., Lescure FX., Daneluzzi V., Vidal B., Clavel G., \& al. (2002). Etude des spondylodiscites infestieuses au CHU d'Amien sur une période de 5 ans. Rev med interne ; 5 (Suppl) : 582s.

22. Broy SB. \& Schmid FR. (1986). A comparison of medical drainage (needle aspiration) and surgical drainage (arthrotomy or arthroscopy) in the initial treatment of infected joints. Clin Rheum Dis; 12 : 50122.

23. Maneiro JR., Souto A., Cervantes EC. \& al. (2015). Predictors of treatment failure and mortality in native septic arthritis. Clin Rheumatol; 34 : 1961-7. 